\title{
Neuropsychological and Linguistic Outcomes after Gunshot Injury to Cerebellum: A Case Report
}

\author{
Mohammed Afsar ${ }^{1} \quad$ Kamasetru Jeevendrakumar Darshini ${ }^{2} \quad$ Dhaval Shukla $^{3}$ Valiyaparambath Vandana ${ }^{2}$ \\ Jamuna Rajeswaran ${ }^{1}$ \\ ${ }^{1}$ Department of Clinical Psychology, National Institute of Mental \\ Health and Neuro-Sciences, Bangalore, Karnataka, India \\ 2Department of Speech Pathology and Audiology, National Institute \\ of Mental Health and Neuro-Sciences, Bangalore, Karnataka, India \\ ${ }^{3}$ Department of Neurosurgery, National Institute of Mental Health

\begin{abstract}
Address for correspondence Jamuna Rajeswaran, MPhil, PhD, Department of Clinical Psychology, National Institute of Mental Health Neuro-Sciences (NIMHANS), 3rd Floor, M V Govindaswamy Centre, Bangalore 560029, Karnataka, India (e-mail: drjamunarajan@gmail.com).
\end{abstract} and Neuro-Sciences, Bangalore, Karnataka, India

Indian J Neurotrauma 2022;19:40-43.

\section{Introduction}

Penetrating traumatic brain injury (TBI), commonly caused by firearms such as guns, are called craniocerebral gunshot injuries (CGIs). CGIs are associated with high case fatality rate (95\%) and chronic neurological disabilities in survivors. ${ }^{1,2}$ Though incidence of CGIs in civilian societies is increasing, most studies on outcomes after CGIs come from military and defense settings showing very long-term cognitive impairments in survivors. ${ }^{3}$ The available literature on the outcomes after CGI in civilian population is limited to case reports or case series and relied on clinical evaluation alone. Very few studies have assessed neuropsychological outcomes after CGIs in civilians using comprehensive and standardized assessment batteries. ${ }^{4-6}$ Moreover, large proportion of the CGI patients described in the literature had sustained injury to anterior brain regions, mainly frontal lobes. The outcomes of CGIs to posterior brain regions including cerebellum remains relatively unexplored. published online April 15, 2021
DOI https://doi.org/

$10.1055 / s-0041-1727294$ ISSN 0973-0508

\section{(C) 2021. Neurotrauma Society of India.}

This is an open access article published by Thieme under the terms of the Creative Commons Attribution-NonDerivative-NonCommercial-License, permitting copying and reproduction so long as the original work is given appropriate credit. Contents may not be used for commercial purposes, or adapted, remixed, transformed or built upon. (https://creativecommons.org/licenses/by-nc-nd/4.0/).

Thieme Medical and Scientific Publishers Pvt. Ltd. A-12, 2nd Floor, Sector 2, Noida-201301 UP, India 
Today, cerebellum is widely implicated in regulation of cognitive and affective functioning in humans and lesion to cerebellum are known to result in cerebellar cognitive affective syndrome (CCAS). ${ }^{7}$ CCAS is characterized by executive dysfunction, poor visuospatial cognition, behavioral/affective changes, and language deficits mainly agrammatism and loss of prosody. ${ }^{7}$ In the last three decades CCAS has been studied extensively in patients with stroke, tumors, or neurodegeneration. There is paucity of research on CCAS in patients with traumatic lesion to cerebellum. The impact of a traumatic lesion may be different from nontraumatic ones because of the mechanical forces involved. This case presents the neuropsychological and linguistic outcomes of cerebellar CGI.

\section{Case Report}

Mr. P.K.G., 53 years old, married gentleman, educated up to 12th standard, working as a Police Inspector in Darjeeling, West Bengal, India, sustained a gunshot injury while raiding a gang of drug dealers. The bullet penetrated the suboccipital region and he immediately lost consciousness. He was immediately rushed to hospital. Three hours later, on arrival to hospital, his Glasgow Coma Scale score was 15/15. The computed tomography (CT) scan of brain was performed, and it showed a foreign body in posterior interhemispheric fissure embedded into the parenchyma of right occipital lobe and few bony fragments with intra-axial pneumocephalus in left cerebellum. There were multiple small splinters from bullet injury in left cerebellar, left vermian, and left occipital area centrally along with acute left cerebellar hemorrhage. He underwent right parieto-occipital decompressive craniotomy and removal of the bullet. He was discharged after 3 weeks to home. Since then, the patient experienced neck pain, speech difficulties (feeling pressure while speaking, reduced clarity of speech), and mild forgetfulness for day-to-day things.

Seven months later, he presented to neurosurgery outpatients services of our institute with above-mentioned complaints. He reported minimal improvement since injury, but experienced subjective distress associated with these symptoms. His socio-occupational functioning remained largely unaffected. The past and personal history were nil contributory. His premorbid cognitive functioning was estimated to be in average range based on the history obtained from the patient and caregiver. A detailed neurological exam by the neurosurgeon was normal. A CT scan of the craniovertebral junction revealed to be normal. He was referred for comprehensive neuropsychological as well as speech-language assessment.

\section{Neuropsychological Assessment}

The neuropsychological assessment was performed using NIMHANS Neuropsychology Battery. ${ }^{8}$ This battery has been standardized for Indian population and provides age-, gender-, and education-matched normative data for interpretation of the scores. The battery consisted of 11 tests and covered cognitive domains of speed and attention, executive functions, visuospatial construction, and learning and memory (both verbal and visual). This battery has been widely used in the evaluation of patients with TBI across the country.
The findings from the neuropsychological assessment are presented in - Table 1. Under the cognitive domain of speed and attention he had moderate impairment in motor speed (finger tapping test) and mild impairment in information processing speed (digit symbol test) and sustained attention (digit vigilance test-errors). The high number of errors on tests of sustained attention is indicative of distractibility which could be resulting from poor interference control. Under the domain of executive functions, he had moderate impairment in semantic fluency (animals' names test) and below average performance in both verbal and visual working memory (digit span and spatial span). Visuospatial construction ability was in average range (complex figure test [CFT]-copy).

CFT and Rey's auditory verbal learning test (RAVLT) were used to assess visual and verbal memory, respectively. Immediate recall score and total learning score on RAVLT along with the immediate recall score on CFT are the measures of encoding component of memory. The delayed recall scores on both RAVLT and CFT are the measures of retrieval component of memory. - Table 1 shows that on CFT, he had mild impairments on both encoding (immediate recall) and retrieval (delayed recall, see - Fig. 1). On RAVLT encoding (total learning and immediate recall) is moderately impaired, whereas retrieval is in below average range. It is noteworthy that in both these tests there was no decline of performance from immediate recall to delayed recall, which is indicative of poor memory functioning resulting mainly from impaired ability to encode information.

\section{Speech-Language Assessment}

Frenchay Dysarthria Assessment was used to evaluate the motor speech and it confirmed the presence of mild dysarthria. ${ }^{9}$ The language functions of the patient were evaluated using Hindi adaptation of Western Aphasia Battery (WAB). ${ }^{10}$ Four main language functions assessed by WAB include-spontaneous speech, comprehension, repetition, and naming. A composite score called Aphasia Quotient (AQ) can be taken as the global index of one's language functioning. On WAB his AQ was calculated to be 97.4 indicative of essentially normal language. Among all four subtests of WAB, he scored lowest on naming (91/100). There were mild word finding difficulties noticed during spontaneous speech. Reading and writing were unaffected.

\section{Discussion}

The present case highlights neuropsychological and linguistic outcomes in a patient with CGI to cerebellum. The comprehensive evaluation showed mild deficits in executive functions such as semantic fluency, working memory, poor interference control (evident in impaired sustained attention), and poor encoding of information along with mild dysarthria and mild word finding difficulties. There was no evidence suggesting visuo-perceptual disturbances or changes in personality/affect.

Executive functions are most consistently associated with cerebellum and its lesions. ${ }^{11}$ Even memory deficits in cerebellar 
Table 1 The neuropsychological profile of the patient

\begin{tabular}{|c|c|c|c|}
\hline Tests & Raw scores & Percentiles $^{\mathrm{a}}$ & Description \\
\hline \multicolumn{4}{|l|}{ Speed and attention } \\
\hline Finger tapping test-Right hand & 36.6 & 05 & Moderate impairment \\
\hline Finger tapping test-Left hand & 33.5 & 05 & Moderate impairment \\
\hline Digit symbol test-Time taken & 300 & 10 & Mild impairment \\
\hline Digit vigilance test-Time taken & 503 & 48 & Average \\
\hline Digit vigilance test-Errors & 12 & 12 & Mild impairment \\
\hline Color trails test 1 -Time taken & 61 & 80 & Above average \\
\hline Color trails test 2 -Time taken & 107 & 81 & Above average \\
\hline \multicolumn{4}{|l|}{ Executive functions } \\
\hline Animals names test & 08 & 05 & Moderate impairment \\
\hline Digit span test & $13 / 30$ & 22 & Below average \\
\hline Spatial span test & $13 / 30$ & 22 & Below average \\
\hline Tower of London-Total problems solved & $09 / 14$ & 60 & Average \\
\hline Stroop test & 184 & 35 & Average \\
\hline \multicolumn{4}{|l|}{ Visuospatial construction } \\
\hline Complex figure test-Copy & $34 / 36$ & 40 & Average \\
\hline \multicolumn{4}{|l|}{ Learning and memory } \\
\hline Complex figure test-IR & $09 / 36$ & 7 & Mild impairment \\
\hline Complex figure test-DR & $10 / 36$ & 15 & Mild impairment \\
\hline RAVLT-total learning & $35 / 75$ & 05 & Moderate impairment \\
\hline RAVLT-IR & $07 / 15$ & 05 & Moderate impairment \\
\hline RAVLT-DR & $09 / 15$ & 22 & Below average \\
\hline
\end{tabular}

Abbreviations: DR, delayed recall; IR, immediate recall; RAVLT, Rey's auditory verbal learning test.

aRaw scores obtained by the patient were converted to percentiles using normative data where higher percentile ranks indicate better performance.

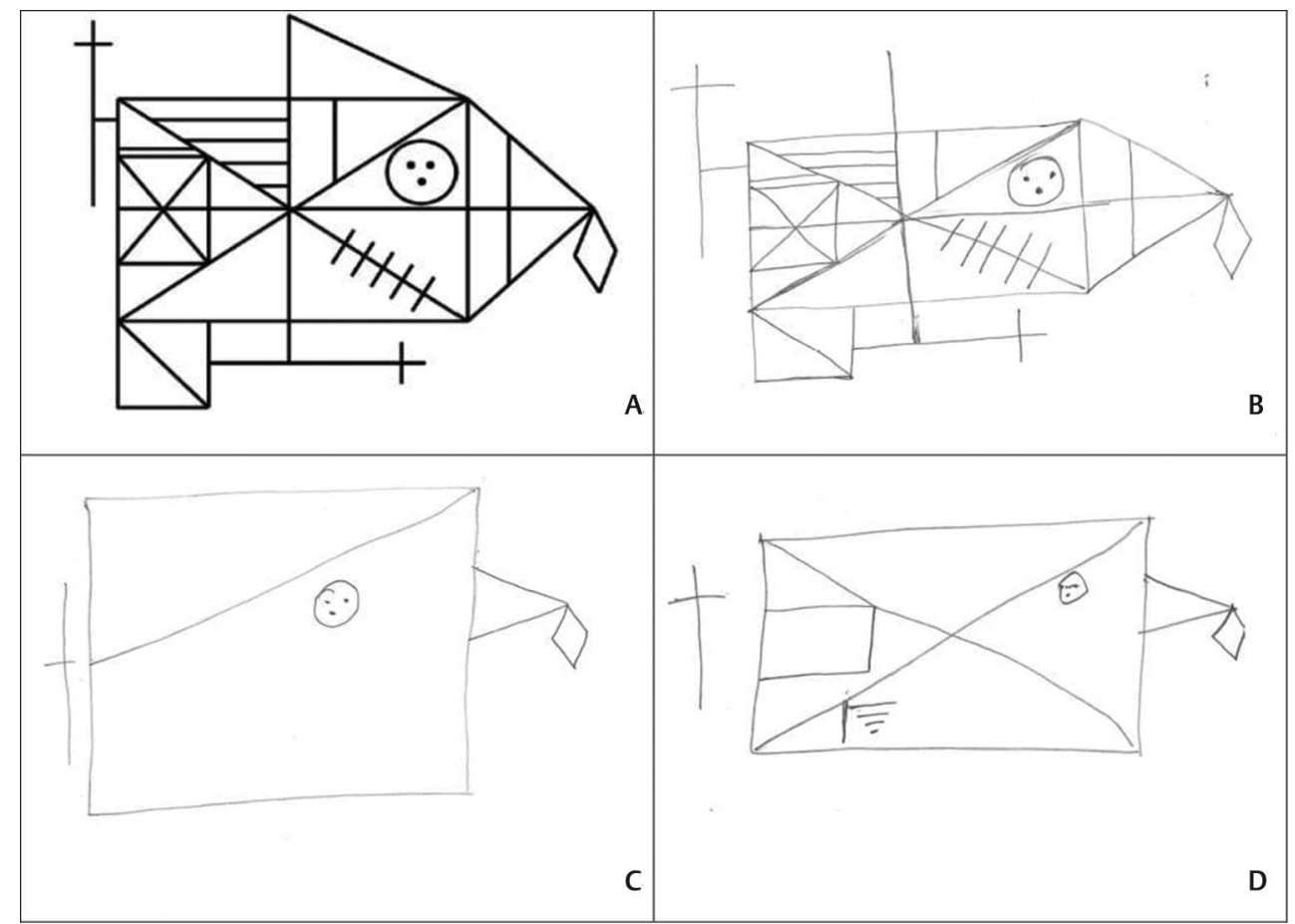

Fig. 1 Patient's performance on test of visuospatial cognition. (A) Stimulus picture for complex figure test, (B) copy of complex figure test done by patient, $(\mathbf{C})$ immediate recall of complex figure test after 3 minutes done by patient, and (D) delayed recall of complex figure test after 30 minutes done by patient. 
diseases appear secondary to executive dysfunction. ${ }^{2}$ Loss of prosody, agrammatism, anomia, reduced word fluency, and ataxic dysarthria are commonly reported speech-language deficits after cerebellar lesion and right-sided lesions producing more severe disturbances. ${ }^{13}$ The CCAS provides a clinical framework to integrate the large body of work surrounding the cognition and affect in cerebellar diseases. CCAS after gunshot injury has not been reported till date.

The profile of neuropsychological and linguistic functioning in our patient is partly confirming to the original description of CCAS. Mild executive dysfunction, mild dysarthria, and mild word finding difficulties are in line with CCAS. However, unlike the original description there was no evidence of agrammatism, loss of prosody, personality, or affective changes, and impaired visuospatial cognition. This partial congruence can possibly be explained by size of lesion, hemisphere involved, and the duration of illness. First, more prominent cognitive and affective disturbances are associated with large, bilateral cerebellar lesions. ${ }^{14}$ Moreover, prominent language disturbances are more commonly associated with damage to right hemisphere of cerebellum. ${ }^{13}$ The cognitive neuroscience literature suggests that the right posterior cerebellar region is implicated in word fluency and category switching during fluency tasks. ${ }^{15}$ Our patient had sustained damage predominantly to the left side of cerebellum which could be the reason for a milder intensity of the symptoms in our patient. Second, cognitive affective disturbances arising from acute cerebellar lesions, for example, stroke, improve within weeks. ${ }^{7}$ Our patient presented after 7 months of injury and no prior evaluation of cognitive, affective, and linguistic functions was documented. We hypothesize that his profile with mild to moderate deficits in neurocognitive and linguistic functioning can be taken to represent partial recovery from CCAS. The persistent residual deficits may point to limited role of spontaneous recovery and warrants for the longitudinal studies.

\section{Conclusion}

The case illustrates the presence of neuropsychological and linguistic disturbances in a patient with cerebellar CGI without any motor or vestibular dysfunctions. The cognitive, affective, and speech language disturbances can induce subjective distress in patients. This case also points to the need for routine clinical screening for cognitive, affective, and language disturbances in patients with cerebellar lesion by treating clinicians. Patients can be referred to specialists if a more comprehensive evaluation is warranted.

\section{Conflict of Interest}

None declared.

\section{References}

1 Shaffrey ME, Polin RS, Phillips CD, Germanson T, Shaffrey CI, Jane JA. Classification of civilian craniocerebral gunshot wounds: a multivariate analysis predictive of mortality. J Neurotrauma 1992;9(Suppl 1) :S279-S285

2 Joseph B, Aziz H, Pandit V, et al. Improving survival rates after civilian gunshot wounds to the brain. J Am Coll Surg 2014;218(1):58-65

3 Dikmen SS, Corrigan JD, Levin HS, Machamer J, Stiers W, Weisskopf MG. Cognitive outcome following traumatic brain injury. J Head Trauma Rehabil 2009;24(6):430-438

4 Kapur N, Hutchinson P, Berry E, Hawkins K, Llewellyn D, Wilson B. Executive dysfunction in a case of transoral-frontal self-inflicted gunshot injury. Brain Inj 2009;23(12):985-989

5 Seniow J, Polanowska K, Mandat T, Laudanski K. The cognitive impairments due to the occipito-parietal brain injury after gunshot. A successful neurorehabiliation case study. Brain Inj 2003;17(8):701-713

6 de Oliveira-Souza R, Moll J, Moll FT, de Oliveira DL. Executive amnesia in a patient with pre-frontal damage due to a gunshot wound. Neurocase 2001;7(5):383-389

7 Schmahmann JD, Sherman JC. The cerebellar cognitive affective syndrome. Brain 1998;121(Pt 4):561-579

8 Rao SL, Subbakrishna DK, Gopukumar K, NIMHANS Neuropsychology Battery. Bangalore, India: National Institute of Mental Health and Neurosciences; 2004

9 Enderby P, Frenchay Dysarthria Assessment. California: College-Hill Press; 1983

10 Karanth P, Western Aphasia Battery in Hindi. Mysore, India: ICMR Project, All India Institute of Speech and Hearing; 1980

11 Baillieux H, De Smet HJ, Paquier PF, De Deyn PP, Mariën P. Cerebellar neurocognition: insights into the bottom of the brain. Clin Neurol Neurosurg 2008;110(8):763-773

12 Appollonio IM, Grafman J, Schwartz V, Massaquoi S, Hallett M. Memory in patients with cerebellar degeneration. Neurology 1993;43(8):1536-1544

13 Schmahmann JD. The cerebellum and cognition. Neurosci Lett 2019;688:62-75

14 Manto M, Mariën P. Schmahmann's syndrome - identification of the third cornerstone of clinical ataxiology. Cerebellum Ataxias 2015;2(1):2

15 Arasanz CP, Staines WR, Roy EA, Schweizer TA. The cerebellum and its role in word generation: a cTBS study. Cortex 2012;48(6):718-724 